\title{
Evaluation of the Accuracy of ADAS Module Readings Based on an Analysis of the Transient Supply Current and Neural Network Application
}

\author{
Damian Grzechca, Pawel Rybka, Krzysztof Paszek \\ Faculty of Automatic Control, Electronics and Computer Science, \\ Silesian University of Technology, \\ 16 Akademicka Street, 44-100 Gliwice, Poland \\ damian.grzechca@polsl.pl
}

\begin{abstract}
The following paper presents advanced methods for evaluating the reliability of ADAS module readings, based on an analysis of the transient supply current. Changes in the transient current waveform occur due to environmental conditions and damage to a module's inner circuitry. Specific deviations in the waveforms may indicate a certain event either internal or external. This paper presents how to successfully distinguish certain anomalies using artificial neural network-based classification algorithms without having to interfere with the module's internal circuitry.
\end{abstract}

Index Terms-Advanced driver assistance systems; Artificial neural networks; Current measurement; Predictive maintenance.

\section{INTRODUCTION}

Advanced Driver Assistance Systems (ADAS) [1] represent one of the fastest growing areas among numerous automotive technologies. They not only improve driving comfort, but what is even more important - they positively contribute to the avoidance of accidents or the mitigation of effects of collisions. Additionally, development of ADAS systems has hastened the achievement of the operation of autonomous vehicles. Consequently, a need to perform research, experiments and full-scale testing in order to validate these novel technologies and to evaluate whether vehicles equipped with such systems can safely operate on public roads and whether the technology itself performs according to specifications has been observed [2]. Carrying out such advanced assessments is not only dictated by the evolving complexity of ADAS, but also by growing requirements of consumers and governmental regulations, which push for higher safety [3] and lower costs [4]. Sensors such as RADARs, LIDARs or cameras play key roles in automotive applications but are vulnerable to environmental conditions and damage to internal circuitry [5], [6]. Although the uncertainty of particular sensors readings can be compensated for by using sensor fusion solutions (Fig. 1), calculations that are based on the data from damaged sensors may unreliable [7].

Manuscript received 11 November, 2017; accepted 19 March, 2018.

This work was supported by the Polish Ministry of Science and High Education funding for statutory activities.

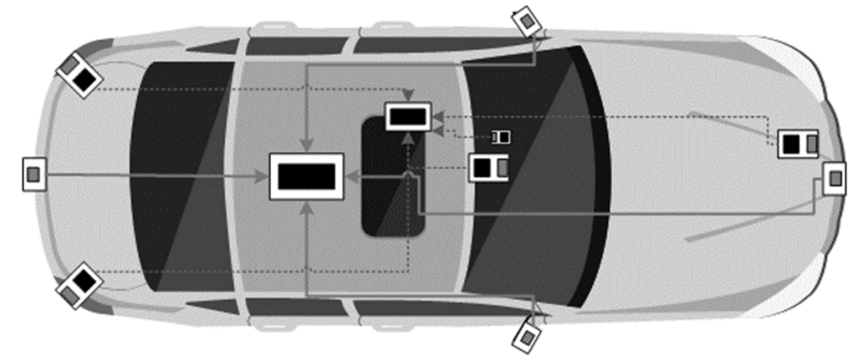

Fig. 1. A conceptual representation of ADAS sensor fusion - a distributed system with data processing in the sensor modules and decision making in the central control unit.

To maximise the reliability of readings, an additional fault-detection method has to be developed to warn the parent system of the possibility of damage to a specific sensor. As ADAS systems are being developed on a day-today basis in automotive R\&D departments worldwide, the method must be versatile and should apply to the vast majority of products. The possibility of implementing the method externally (without having to interfere with the integrity of a certain module's hardware) would also be a great step forward when considering the development of novel sensors - engineers would not have to implement the solution in every sensor that is released. Such a versatile quality-monitoring tool has to be based on a set of information that would describe the current state of the sensor without interfering with its inner structure. This can be done by examining the values of the module's temperature in particular spots, analysing the supply current waveforms [8] for certain voltage levels or measuring the timing and noises on the communication line.

The method for enhancing the reliability of the readings of ADAS sensors that are presented in this article is based on the analysis of transient current waveform using heuristic methods of classification. The data, after being extracted, is processed by both a supervised learning backpropagation algorithm [9] and an SOM (Self-organising Map) [10].

\section{DATA ACQUiSITION}

The first step in developing a reliability assessment module is collecting the essential data concerning operational conditions the ADAS sensors - in this case the 
emphasis will be put on the current measurements. There are numerous ways to acquire signals for the transient supply current waveforms. The most popular of these are using an analogue-to-digital converter (ADC) [11] or a microcontroller that is interconnected with either a Hall sensor [12] or an instrumentation amplifier with shunt resistance (Fig. 2). To obtain a representative transient current waveform, the signal has to be sampled at a certain frequency from the moment it is turned on until it enters a steady state. When the samples are collected, they are ready to be sent for further analysis.

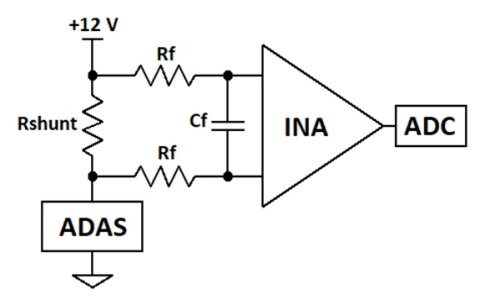

Fig. 2. Transient current waveform acquisition circuit for an ADAS output reliability assessment module - instrumentation amplifier with a shunt resistor and filter.

The module, which is built for the reliability assessment of ADAS sensors, samples the signal at a frequency of $100[\mathrm{kHz}]$ for around $70[\mathrm{~ms}]$ (to ensure a steady state is reached for any device). This provides a total of 7000 samples - enough for further classification. The transient current waveforms for four representative ADAS modules are shown below (Fig. 3).

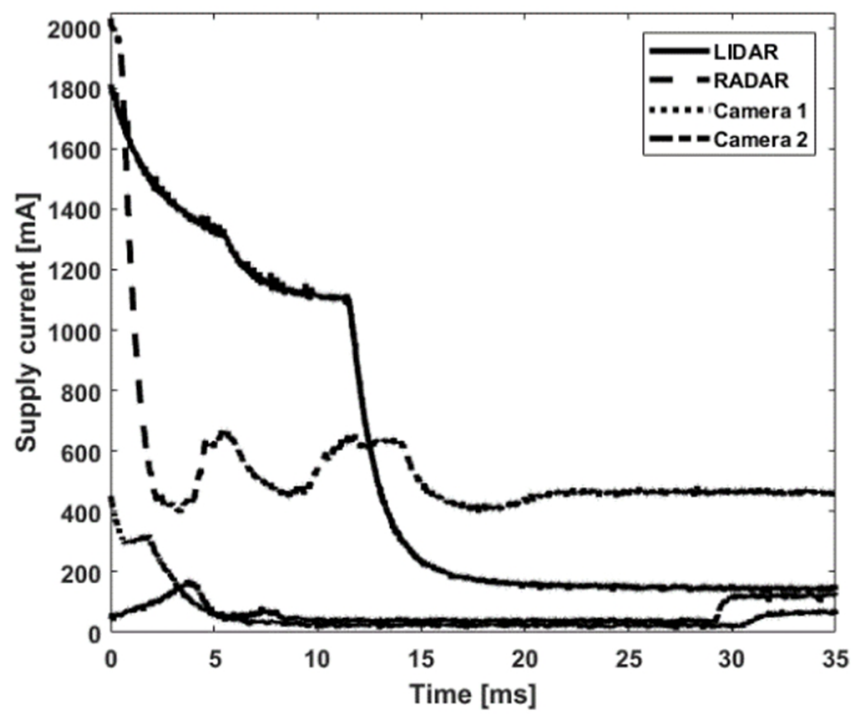

Fig. 3. Transient current waveforms of the specific ADAS sensors that were obtained by the module.

At first glance, it can be seen that those waveforms differ from one another. Those differences will allow for the further classification and examination of what type of device is connected to the assessment module and under what conditions it operates.

\section{SIGNAL PRE-PROCESSING}

The acquired signal consists of 7000 samples - too many to use for classification. Moreover, the signal is typically collected in an environment that is heavily exposed to external sources of noise, e.g. a vehicle's electrical installation. Those reasons require additional steps for the processing of the waveforms before it can be fed into the input of the classifying algorithm. The first step to be taken is filtering the input signal from the environmental noise. Such filtration can be performed using any low-pass filter, e.g. by the moving average filter (1) [13] with a window size wide enough to smooth the signal, while on the other hand, narrow enough not to distort it. The given filter smoothens the signal by calculating the mean value of a fixed number of its neighbours (window width equals $2 \mathrm{~N}$ )

$$
f_{s}(x)=\frac{1}{2 N+1} \sum_{i=-[N]}^{[N]} f(x+i) \text {. }
$$

For a transient supply current waveform, a window width of 20 ensures enough signal filtration without having to worry about any distortion. The filtrated data is ready for the next pre-processing step - extraction. Classification methods, especially heuristic one, require an accurate selection of their input data for the maximisation of classification precision. There are plenty of methods for selecting the most valuable features from a signal - the ones that are used most frequently are the Naive Bayes classifier, numerous genetic algorithms, the Fourier or other transforms. By using the wavelet transform [14], the signal can be described with a set of approximation and detail coefficients. From each whole waveform (composed of 7000 samples), the $7^{\text {th }}$ level Haar wavelet [15] transformation delivers 25 approximation coefficients, which can be used as the input for classification algorithm. The following discrete sequence plot shows how a LIDAR's waveform changes (caused by varying amount of supply voltage) affect the transformation coefficients (Fig. 4).

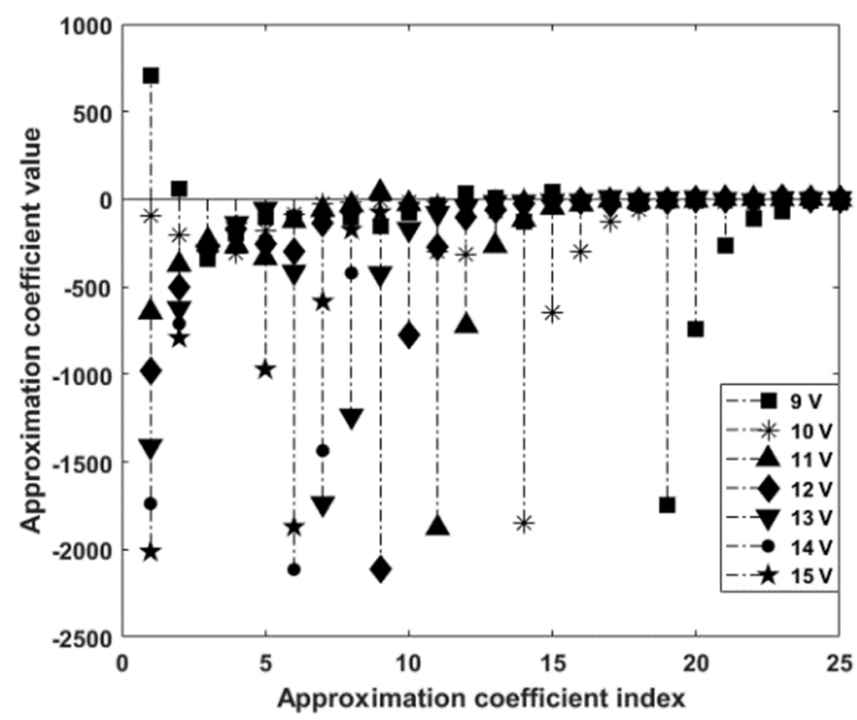

Fig. 4. Approximation coefficient values of LIDAR's $7^{\text {th }}$ level wavelet transformation obtained for various supply voltage levels.

In the figure above, it can be clearly seen that even slight changes in the supply voltage can cause significant changes in the values of the wavelet transformation coefficients. This ensures that the classification input vector will be sensitive to even the smallest signal deviations, which is crucial for the reliability estimation system - each slight change in the environment or inner circuitry can be detected.

Another method for extracting valuable data, which can be used for transient current analysis, is based on selecting 
the most valuable samples rather than complex signal processing. The process, which is called naive Bayesian classification [16] - equation (2), shows the probabilities a model's posterior and can be used to select samples that tend to show the highest potential for successful classification. To minimise the possibility of affecting the sample selection by noise, a window that would filter out individual well-performing samples can be applied when their neighbour's quality is poor. Such a filter causes only consecutive well-matched features to be taken into account

$$
\begin{gathered}
\hat{P}\left(Y=k \mid X_{1}, \ldots, X_{p}\right)= \\
=\frac{\pi(Y=k) \prod_{j=1}^{P} P\left(X_{j} \mid Y=k\right)}{\sum_{k=1}^{K} \pi(Y=k) \prod_{j=1}^{P} P\left(X_{j} \mid Y=k\right)},
\end{gathered}
$$

where $\mathrm{Y}$ - random variable corresponding to the class index of an observation; $\mathrm{Xj}$ - predictor of an observation; $\pi(\mathrm{Y}=\mathrm{k})$ - prior probability that the class index is $\mathrm{k}$.

The algorithm searches for samples (or rather series of samples) whose probability of the correct classification of this single feature is settled above a fixed threshold. When this collection of samples is acquired, it has to be decimated in order to obtain an input vector for the classification of a certain length. The plot below (Fig. 5) shows a transient current waveform with the set of samples that was selected by the naive Bayesian pre-classification (upper graph) and its decimated subset (lower graph). The probability threshold $\mathrm{p}=75 \%$, window width $\mathrm{w}=10$ (341 samples). The subset is decimated to 27 samples, which are then fed into the classification algorithm's input.

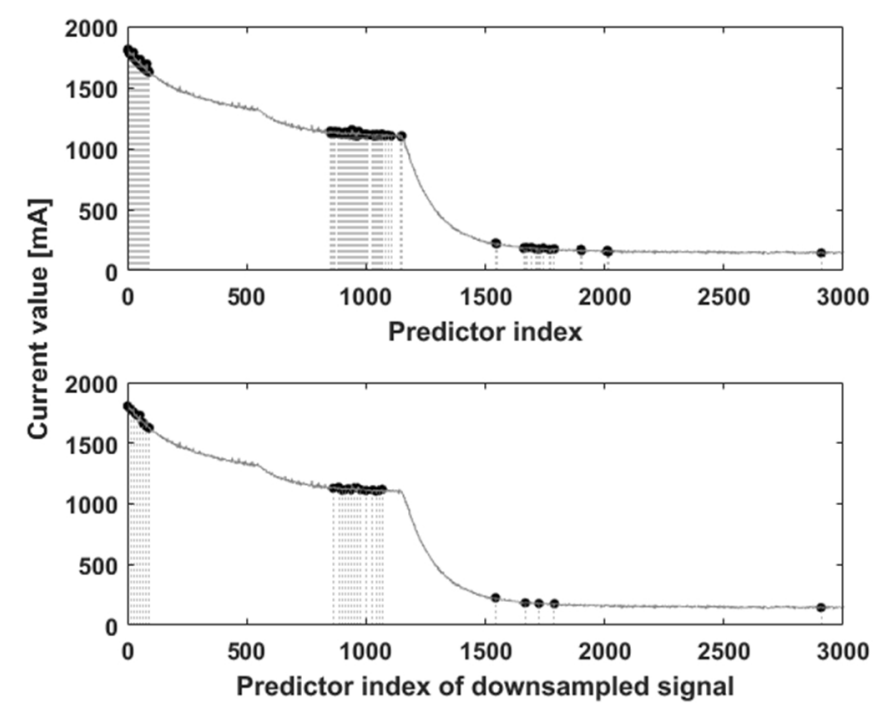

Fig. 5. LIDAR transient current waveform with the set of samples that was selected by the naive Bayesian algorithm (upper) and its decimated subset (lower).

The last extraction method to be presented is the simple decimation of the original waveform (3). It does not provide any enhancement for further classification and therefore it is expected to bring the worst results from all of the preprocessing methods

$$
f_{d}(x)=f(n x)
$$

The decimation coefficient $\mathrm{n}$ is selected in such a manner that the number of features after applying the decimation is equal 25 (for 7000 samples $n=280$ ). A comparison of the naive Bayesian pre-classification with the decimation (upper graph) and a regular decimation (lower graph) method for RADAR sensor is shown in the following plot (Fig. 6).

The main role of the decimation method is to compare the two previous approaches and to assess the importance of feature extraction related to classification issues.

As all of the pre-processing methods are presented, validation of their efficiency will be achieved after feeding the outputs into certain classification algorithms and comparing their performance.

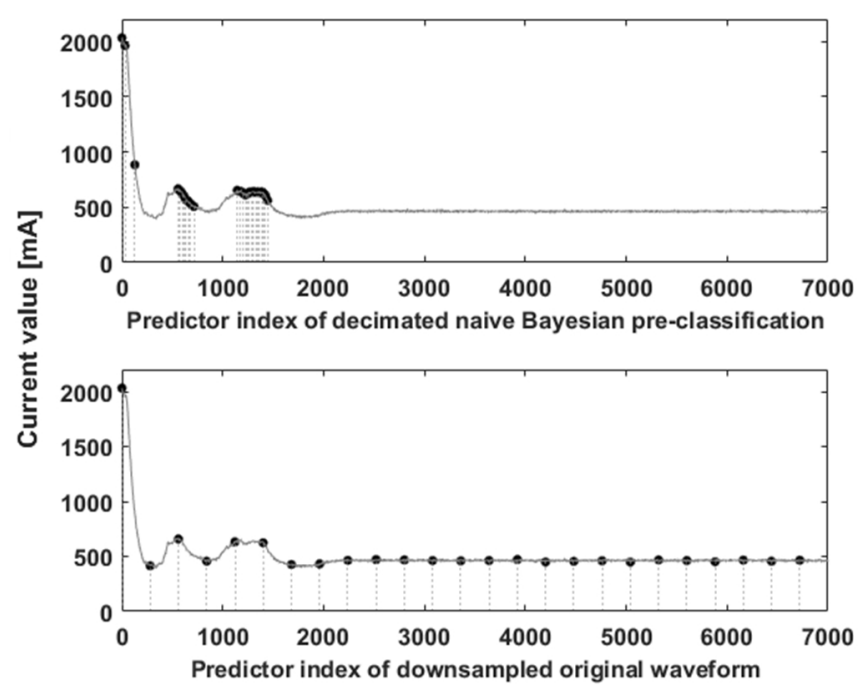

Fig. 6. RADAR transient current waveform with the set of samples that was elected by the decimated naive Bayesian algorithm (upper) and the original decimated signal (lower).

\section{SIGNAL CLASSIFICATION}

All of the classification algorithms used in this paper are based on machine learning - either simple, e.g. k-Nearest Neighbours (k-NN) [17] or more complex - SOM or feedforward backpropagation artificial neural network. Before analysing any transient current changes due to voltage variation, the first step that has to be performed is examining what kind of device is connected to the acquisition circuit. As the signals of all of the ADAS sensors differ significantly from one another, there is no need to implement advanced pre-classification techniques. The best and easiest method to implement is SOM. This algorithm creates a map of the classified signals without the need to provide the expected output vector (unsupervised learning). The $5 \times 5$ map that is created using 520 decimated (by the factor $n=280$ ) current waveforms of four ADAS sensors is presented below (Fig. 7).

It can be observed that all of the waveforms were classified correctly - there are no mismatches in any of the four groups and what is worth noting is that the clusters are separated by at least one cell, which ensures an even better signal grouping. Another part that is visible at first glance is the fact that the classification of the set of waveforms for selected module is divided among several cells. This 
phenomenon is caused by the fact that for 130 transient current waveforms of a certain module, every set of ten signals was obtained for a different supply voltage level (from $9 \mathrm{~V}$ to $15 \mathrm{~V}$ with a step of $0.5 \mathrm{~V}$ ).

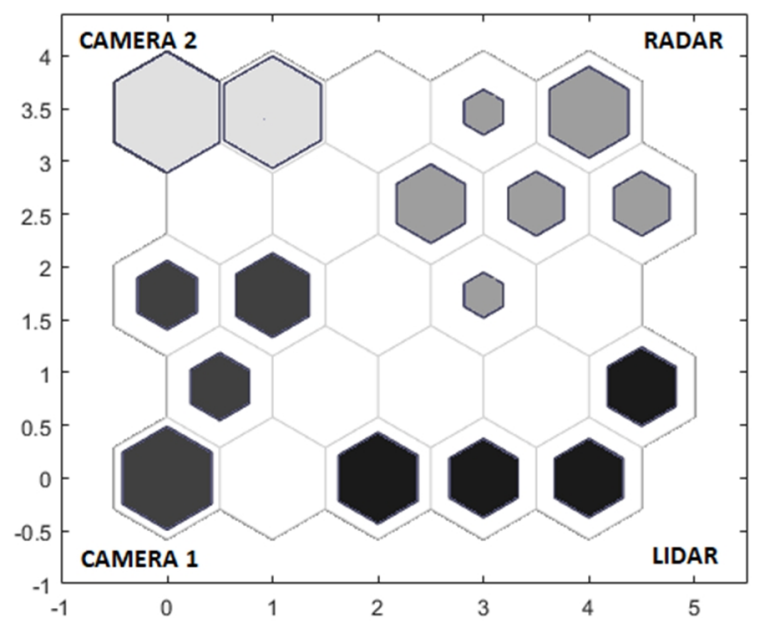

Fig. 7. Classification of ADAS sensors using the SOM method - map of sample hits.

As soon as a device that is connected to the transient current acquisition circuit is classified, there is another classifier to be trained to distinguish at which voltage the module is operating. When the voltage over a certain sensor is measured and the output of a certain classifier is known comparing those two values can provide knowledge about whether the sensor is working correctly and whether the data it is providing is reliable.

The first algorithm to be tested is k-NN. The method is trained with a set of waveforms of a particular sensor (RADAR) - ten samples for each voltage level from $9 \mathrm{~V}$ to $15 \mathrm{~V}$ with a step of $0.5 \mathrm{~V}$ (130 samples in total). The validation waveforms are generated using dedicated modelling software - signals will vary from $10 \mathrm{~V}$ to $14 \mathrm{~V}$ (Fig. 8).

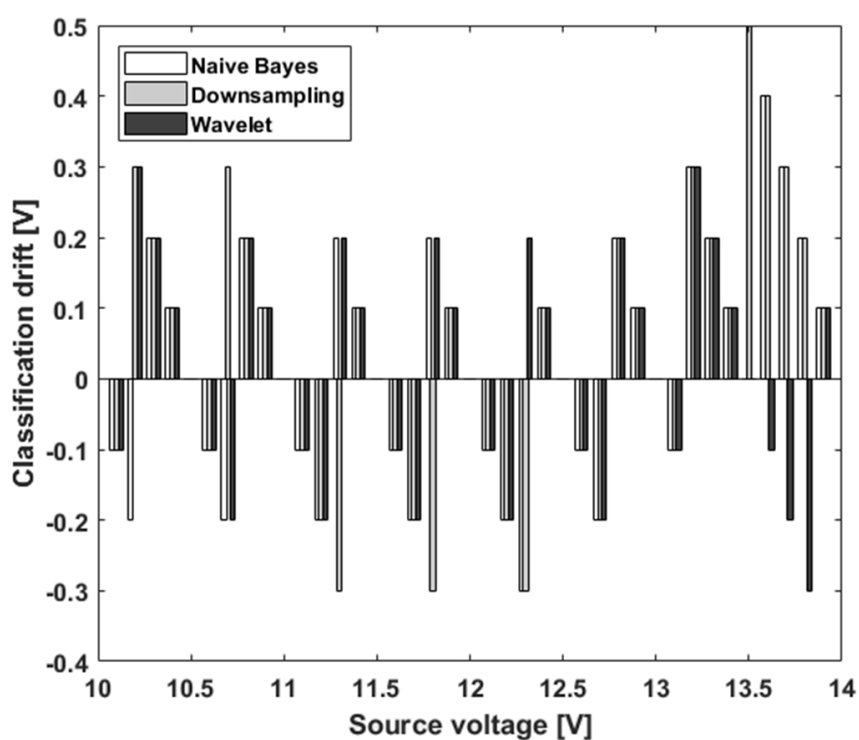

Fig. 8. Classification of the voltage level using the k-NN algorithm and different pre-classification methods.

It can be seen that the misclassification is the highest for a source voltage above $13 \mathrm{~V}$. This may be caused by low signal change dynamics at this voltage range. The k-NN algorithm is characterised by the fact that it cannot distinguish voltage changes that are different from the ones that it was prepared to classify. This means that for any voltage supply that is different from $10 \mathrm{~V}$ to $14 \mathrm{~V}$ with a step of $0.5 \mathrm{~V}$, it will give additional error, which is not dependent on misclassification issues, but rather on the inner algorithm structure. That is the main disadvantage of using such a method.

Based on the Table I, it can clearly be seen that using the wavelet transform's approximation coefficients gives the best classification results for the $\mathrm{k}-\mathrm{NN}$ algorithm. The second best is the naive Bayes method and the worst - as expected - the simple signal decimation.

Another approach to the classification problem is the use of the artificial neural network backpropagation algorithm. Such an algorithm has an advantage over k-NN as it can distinguish any voltage from certain bounds and can classify it not only on certain subgroups with $0.5 \mathrm{~V}$ step. Such a neural network can be trained in very different ways. From various the backpropagation networks that can used (feedforward, Elman and cascade-forward), the best output was found for the cascade-forward with five hidden neurons. For the naive Bayes, the vector has 27 elements and for the downsampling signal, it has 26 elements - the vectors' lengths were selected arbitrarily during multiple experiments. For the wavelet transform, the length was set to 25 elements, which is based on the transform's nature. One element output vector represents the actual analog input voltage level value.

The neural network trained with the same sets of data that was used for the k-NN algorithm gave the voltage drift results that are presented in Fig. 9.

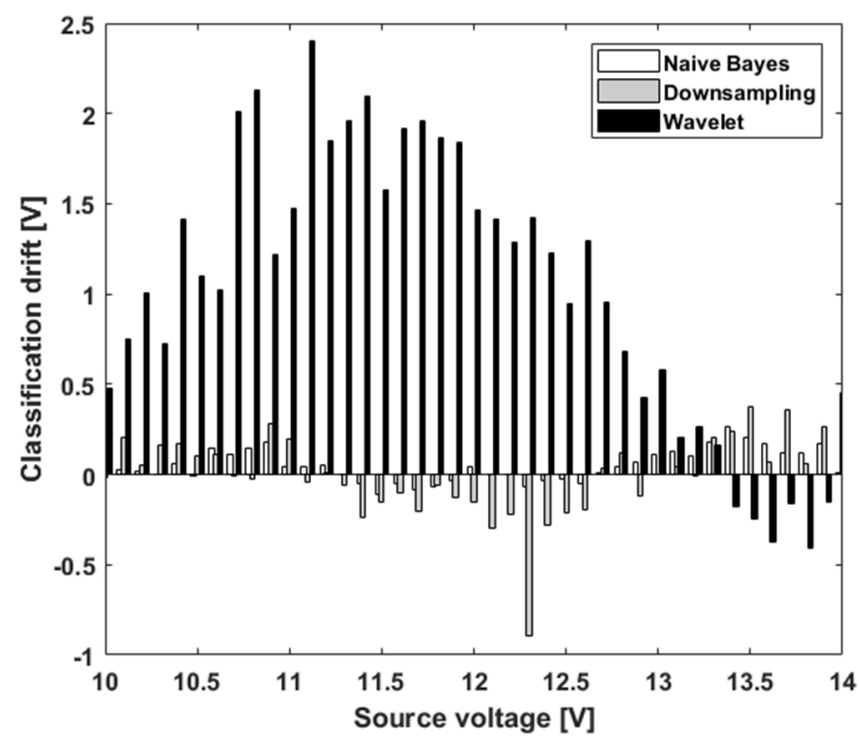

Fig. 9. Identification of the voltage level using the cascade-forward backpropagation neural network and different pre-classification methods.

Surprisingly, the input vector of the wavelet approximation coefficient values provided the poorest result - this may be caused by its high sensitivity to minor changes in the waveforms on which the neural network is based. At voltage source values greater than $13 \mathrm{~V}$, the change dynamics are not significant compared to the rest of the signal and the wavelet transform's error is thus small (below $0.5 \mathrm{~V})$. On the other hand, the naive Bayes pre-classification 
algorithm performed much better than the k-NN and gave the best match of all. The simple decimation gave a similar average error as in k-NN, but the maximum error of almost $0.9 \mathrm{~V}$ disqualified it from being a reliable source for assessing ADAS sensors. The comparison of those values is presented in Table I below - the best match is written in italics.

TABLE I. K-NN CLASSIFICATION AND NEURAL NETWORK IDENTIFICATION PERFORMANCE COMPARISON.

\begin{tabular}{|c|c|c|c|}
\hline Algorithm & $\begin{array}{c}\text { Pre-classification } \\
\text { algorithm }\end{array}$ & $\begin{array}{c}\text { Maximum } \\
\text { error [V] }\end{array}$ & $\begin{array}{c}\text { Average } \\
\text { error [V] }\end{array}$ \\
\hline K-NN & Decimation & 0.5 & 0.15 \\
\hline K-NN & Naive Bayes & 0.4 & 0.13 \\
\hline K-NN & Wavelet & 0.3 & 0.12 \\
\hline Neural network & Decimation & 0.89 & 0.16 \\
\hline Neural network & Naive Bayes & 0.26 & 0.07 \\
\hline Neural network & Wavelet & 2.39 & 1.10 \\
\hline
\end{tabular}

The table shown above represents the performance of different algorithms (including pre-classification as well) of the entire signal domain. To examine those processes more deeply, such analysis should also be performed on the subsets of the domain (e.g. to localise the weakest links in the system or to find the cause of such poor behaviour). For such an analysis, two of the best fitting algorithms can be used - neural network with the naive Bayes preclassification and k-NN with wavelet pre-classification. The following Table II compares their error relations for certain subsets (best values are written in italics).

TABLE II. COMPARISON OF ERROR ON THE VOLTAGE SUBSETS FOR THE TWO LEADING METHODS.

\begin{tabular}{|c|c|c|c|c|}
\hline \multirow[b]{2}{*}{$\begin{array}{c}\text { Supply } \\
\text { voltage } \\
\text { range }[\mathrm{V}]\end{array}$} & \multicolumn{2}{|c|}{$\begin{array}{l}\text { Mean classification error } \\
{[\mathrm{mV}]}\end{array}$} & \multicolumn{2}{|c|}{$\begin{array}{c}\text { Max. classification error } \\
{[\mathrm{mV}]}\end{array}$} \\
\hline & $\begin{array}{c}\text { K-NN } \\
\text { (wavelet) }\end{array}$ & $\begin{array}{c}\text { Neural } \\
\text { network } \\
\text { (naive } \\
\text { Bayes) }\end{array}$ & $\begin{array}{c}\text { K-NN } \\
\text { (wavelet) }\end{array}$ & $\begin{array}{c}\begin{array}{c}\text { Neural } \\
\text { network } \\
\text { (naive } \\
\text { Bayes) }\end{array} \\
\end{array}$ \\
\hline $10-10.5$ & 140 & 30 & 300 & 60 \\
\hline $10.5-11$ & 120 & 118 & 200 & 178 \\
\hline $11-11.5$ & 120 & 40 & 200 & 53 \\
\hline $11.5-12$ & 120 & 70 & 200 & 110 \\
\hline $12-12.5$ & 120 & 29 & 200 & 65 \\
\hline $12.5-13$ & 120 & 38 & 200 & 72 \\
\hline $13-13.5$ & 133 & 157 & 300 & 266 \\
\hline $13.5-14$ & 131 & 131 & 300 & 266 \\
\hline
\end{tabular}

Based on the table above, it can be seen that apart from the mean errors in the subset from $13 \mathrm{~V}-14 \mathrm{~V}$, the neural network with the naive Bayes feature extraction algorithm performs better than k-NN with a wavelet pre-classification. What can be seen as well is the fact that both algorithms perform best in the middle of the domain $(11 \mathrm{~V}-13 \mathrm{~V})$ and worse when reaching the voltage boundaries. This behaviour is completely acceptable from the automotive usage point of view, as during the vast majority of time, the voltage does not strongly vary from $12 \mathrm{~V}$. The crucial part of the received data (maximum error) can also be shown on a bar plot (Fig 10).

Both classification methods provided output that was sufficient enough to be used in evaluating ADAS' modules readings. Moreover, in order to enhance the classification, both methods can be used simultaneously - complementary to each other. Combining two or even more methods, which are highly different from one another, increases the certainty of a final classification - each method can be sensitive to different factors (e.g. temperature or aging). Such a solution would require the use of some kind of data fusion method e.g. the Kalman filter. A schematic diagram of such merging would look like that below (Fig. 11).

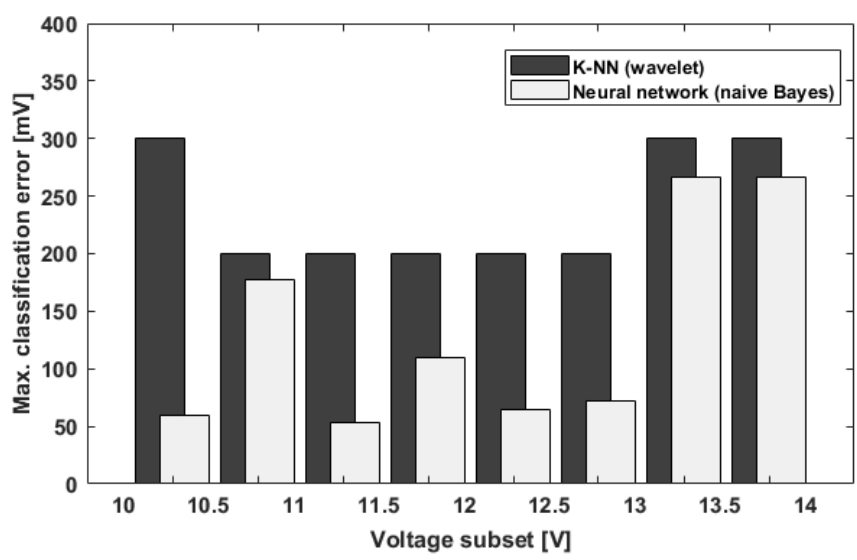

Fig. 10. Maximum classification voltage drift for k-NN with the wavelet transformation algorithm and a neural network with the naive Bayes pre-classification.

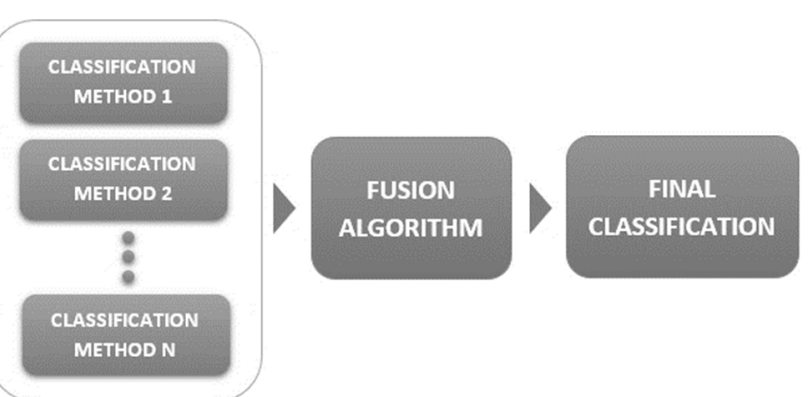

Fig. 11. Graphical representation of merging of classification data.

The data processed in such a manner is resistant to the uncertainty of a particular method, which results in a higher reliability of the classification data. The final output in such a shape is ready to be fed into the last section - the ADAS the assessment module for the sensor readings.

\section{EVAluation OF THE RELIABILITy OF SENSOR READINGS}

Having developed the classification system, the final step that is required to successfully assess the reliability of the ADAS sensor readings is to insert a received current waveform of a specific module into the classification algorithm. When the output data that is related to its environment (operating temperature, supply voltage level, aging) is generated by the algorithm based on a signal, the next step is to compare those values to the real-world ones. Based on this comparison, the accuracy level of a reading's reliability for a certain sensor can be determined - the current waveform of a damaged sensor would behave differently than an intact one. A block diagram of such an approach is presented in (Fig. 12)

The comparison algorithm block represents a function that establishes a reading's reliability based on the difference between the real-world data (e.g. data acquired by a voltmeter or thermometer) and the classification data voltage or temperature based on an input waveform. The bigger the difference for a certain feature - the lower the 
reading's reliability should be.

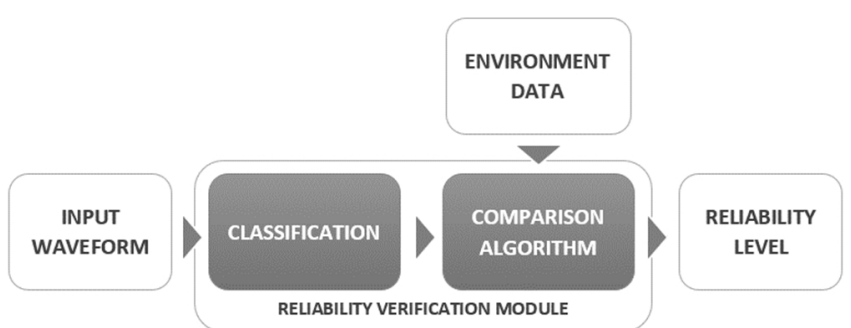

Fig. 12. Conceptual diagram of the assessment of the reliability level for ADAS sensor readings.

To assign a certain reliability level to a particular current waveform, a function that converts the deviation to a percentage reliability value has to be developed. Such a function can be, for example, linear (4), Gaussian (5) or window (6):

$$
\begin{gathered}
f(x)=\left\{\begin{array}{ccc}
|n x|+1, & \text { for } & |x| \leq \frac{1}{a}, \\
0, & \text { for } & |x|>\frac{1}{a},
\end{array}\right. \\
f(x)=e^{-\left(\frac{n x^{2}}{2}\right)}, \\
f(x)=\left\{\begin{array}{lll}
1, & \text { for } & |x| \leq n, \\
0, & \text { for } & |x|>n .
\end{array}\right.
\end{gathered}
$$

Based on the equations presented above, a reliability assessment plot can be created (Fig. 13). With a change of coefficient $\mathrm{n}$ in the equations, a certain plot can be adjusted for a specific sensor (each ADAS module's behaviour is different and the choice of a method and its width strictly depends on its type).

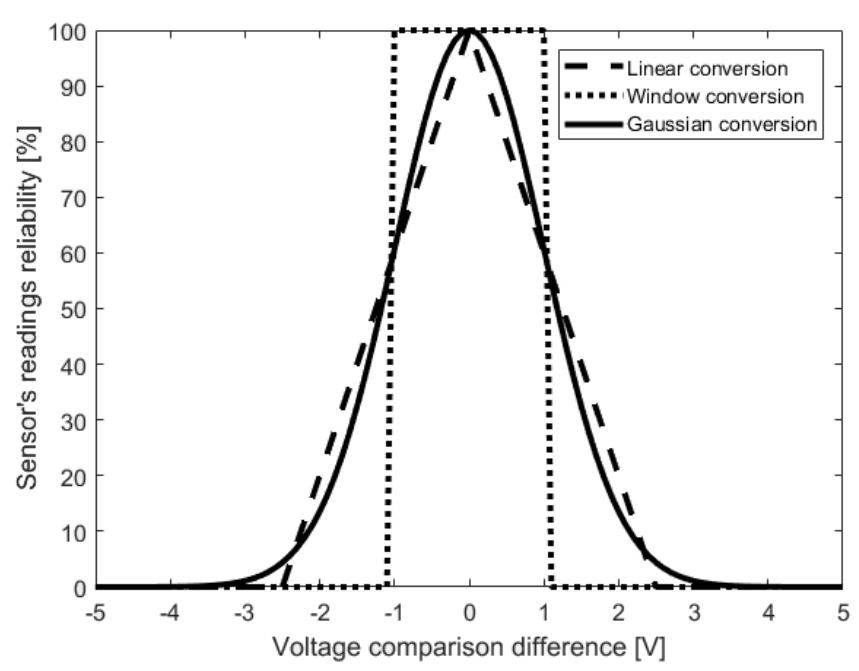

Fig. 13. Comparison of different conversion curves.

For the curves shown above (for linear conversion $n=0.4$, for window $n=1$, for Gaussian $n=1$ ), the reliability of exemplary reading for specific voltage drifts are shown in Table II.

Each of the presented methods has its advantages and disadvantages - the decision of which method to choose should be based on the needs of a particular application.
TABLE III. RELIABILITY OF THE READINGS FOR CERTAIN CONVERSION CURVES.

\begin{tabular}{|c|c|c|c|}
\hline \multirow{2}{*}{$\begin{array}{c}\text { Voltage drift } \\
\text { [V] }\end{array}$} & $\begin{array}{c}\text { Linear } \\
\text { conversion } \\
(\mathbf{n}=\mathbf{0 . 4})\end{array}$ & $\begin{array}{c}\text { Window } \\
\text { conversion } \\
(\mathbf{n}=\mathbf{1})\end{array}$ & $\begin{array}{c}\text { Gaussian } \\
\text { conversion } \\
(\mathbf{n}=\mathbf{1})\end{array}$ \\
\hline-1.5 & 40 & 0 & 33 \\
\hline-1 & 60 & 100 & 61 \\
\hline-0.5 & 80 & 100 & 88 \\
\hline 0.1 & 96 & 100 & 99 \\
\hline 0.7 & 72 & 100 & 78 \\
\hline 2 & 20 & 0 & 14 \\
\hline
\end{tabular}

\section{CONCLUSIONS}

The presented paper has shown that the evaluation of accuracy of an ADAS module's readings can be based on transient current analysis and the application of a neural network. Moreover, more than one way of classification and pre-classification appears to exist, which shows a method's versatility and creates a field to develop in the future thus enhancing classification methods. For assessing the reliability of an ADAS sensor's readings, the two best methods were the k-NN classification with wavelet transformation as a feature extraction and the cascadeforward backpropagation neural network with the naive Bayes pre-classification algorithm. Those methods, alongside the final conversion curves, have to be selected independently for the needs particular application, as the methodology presented in the paper can also be used in nonautomotive fields as well.

\section{REFERENCES}

[1] A. Ziebinski, R. Cupek, D. Grzechca, L. Chruszczyk, "Review of advanced driver assistance systems (ADAS)", in Proc. AIP Conf., 2017. DOI: 10.1063/1.5012394.

[2] K. Tokarz, J. Paduch, L. Herb, "Influence of Receiver parameters on GPS navigation accuracy", Man-Machine Interactions, vol. 2, no. 103, pp. 85-93, 2011. DOI: 10.1007/978-3-642-23169-8_10.

[3] B. Fildes, M. Keall, P. Thomas, K. Parkkari, L. Pennisi, C. Tingvall, "Evaluation of the benefits of vehicle safety technology: The MUNDS study", Accident Analysis \& Prevention, vol. 55, pp. 274 281, 2013. DOI: 10.1016/j.aap.2013.02.027.

[4] M. Drewniak, K. Tokarz, M. Redzinski, "ADAS device operated on CAN bus using PiCAN module for raspberry Pi", Computational Collective Intelligence, Lecture Notes in Computer Science, vol. 10449, pp. 227-237, 2017. DOI: 10.1007/978-3-319-67077-5 22.

[5] K. Budniak, K. Tokarz, D. Grzechca, "Practical verification of radio communication parameters for object localization module", ManMachine Interactions, vol. 4, no. 391, pp. 487-498, 2016. DOI: 10.1007/978-3-319-23437-3_41.

[6] D. Grzechca, "Soft fault clustering in analog electronic circuits with the use of self organizing neural network", Metrology and Measurement Systems, vol. 18, no. 4, pp. 555-568, 2011. DOI: 10.2478/v10178-011-0054-8T

[7] A. Ziebinski, R. Cupek, H. Erdogan, S. Waechter, "A survey of ADAS technologies for the future perspective of sensor fusion", Computational Collective Intelligence, vol. 9876, pp. 135-146, 2016. DOI: 10.1007/978-3-319-45246-3_13.

[8] D. Grzechca, A. Ziebinski, P. Rybka, "Enhanced reliability of ADAS sensors based on the observation of the power supply current and neural network application", Computational Collective Intelligence, vol. 10449, pp. 215-226, 2017. DOI: 10.1007/978-3-319-67077-5_21.

[9] J. Sietsma, R. J. F. Dow, "Creating artificial neural networks that generalize", Neural Netw., vol. 4, no. 1, pp. 67-79, 1991. DOI: 10.1016/0893-6080(91)90033-2.

[10] T. Kohonen, "The self-organizing map", Proc. IEEE, vol. 78, no. 9, pp. 1464-1480, 1990. DOI: 10.1109/5.58325.

[11] P. Fexa, J. Vedral, "Developing automated data acquisition system for $\mathrm{ADC}$ and DAC testing", in 6th IEEE Int. Conf. Intelligent Data 
Acquisition and Advanced Computing Systems, 2011, pp. 39-42. DOI: 10.1109/IDAACS.2011.6072707.

[12] S. Francis, I. Ruban, S. Karthikeyan, R. Surenthiranath, S. Jeyadevi, "Coreless Hall effect based current sensing technique with magnetic shielding", 2017.

[13] S. W. Smith, The scientist and engineer's guide to digital signal processing. San Diego, Calif: California Technical Pub, 1997.

[14] L. Chruszczyk, "'Wavelet transform in fault diagnosis of analogue electronic circuits", Advances in Wavelet Theory and Their Applications in Engineering, Physics and Technology, 2012. DOI:
$10.5772 / 36423$.

[15] U. Lepik, H. Hein, "Haar Wavelets", Haar Wavelets, pp. 7-20, 2014. DOI: 10.1007/978-3-319-04295-4 2.

[16] T. Hastie, R. Tibshirani, J. H. Friedman, The elements of statistical learning: data mining, inference, and prediction. New York, NY: Springer, 2009. DOI: 10.1007/978-0-387-84858-7.

[17] T. Cover, P. Hart, "Nearest neighbor pattern classification", IEEE Trans. Inf. Theory, vol. 13, no. 1, pp. 21-27, 1967. DOI: 10.1109/TIT.1967.1053964. 